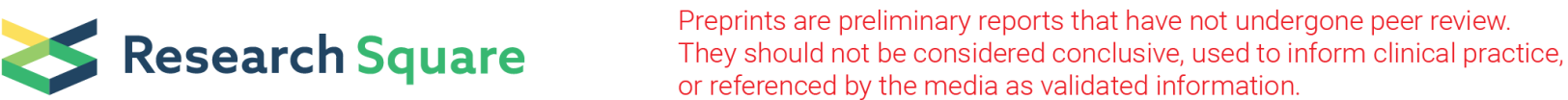

\section{Comparison of Computed Tomographic Imaging- guided hook wire localization and electromagnetic navigation bronchoscope localization in the resection of pulmonary nodules}

\section{Yu Tian}

The Second Hospital of Shandong University

\section{Ming Lu}

School of Medicine, Shandong University

Weiming Yue

Shandong University Qilu Hospital

Libo Si

Shandong University Qilu Hospital

Lin Li

Shandong University Qilu Hospital

Shuhai Li

Shandong University Qilu Hospital

Hui Tian ( $\nabla$ tianhuiql@163.com )

Shandong University Qilu Hospital

Research article

Keywords: electromagnetic navigation bronchoscopy; lung cancer; pulmonary nodule; thoracoscopic surgery

Posted Date: October 4th, 2019

DOI: https://doi.org/10.21203/rs.2.15619/v1

License: (c) (i) This work is licensed under a Creative Commons Attribution 4.0 International License. Read Full License 


\section{Abstract}

Background: The resection of nodules by thoracoscopic surgery is difficult because the nodules may be hard to identify. Currently, preoperative localization of pulmonary nodules is widely used in the clinic, including Computed Tomographic Imaging (CT)-guided transthoracic approach, hook-wire and coil embolization, but these methods increase radiation exposure and cause complications. Methods: In this study, we retrospectively compared electromagnetic navigation bronchoscopy (ENB) guided and CT guided localization of small pulmonary nodules before resection. Results: Total 157 patients underwent the localization procedure successfully, and the nodules were localized by CT guidance in 105 patients and by ENB in 52 patients. The nodule size of magnetic navigation localization was smaller than that of CT-guided localization $(P<0.001)$. Both CT-guided and ENB localization were well tolerated in all patients, without major complications or mortality $(P=0.107)$. In CT-guided localization group, 6 patients failed to be located while none failed in ENB group $(P=0.079)$. The procedure time was $15.15 \pm \llbracket 3.7 \mathrm{~min}$ for CTguided localization and $21.29 \pm \llbracket 4.0 \mathrm{~min}$ for ENB localization $(P<0.001)$. Conclusions: CT-guided localization is simple and feasible for uncertain pulmonary nodules before surgery. ENB localization could identify small lung nodules with high accuracy and low incidence of complications.

\section{Background}

Lung cancer is the most lethal disease worldwide. It accounts for one-quarter of all cancer deaths around the world. Although the diagnosis and treatment technologies have improved rapidly in recent years, the prognosis of lung cancer remains poor. The 5 -year survival rate of lung cancer is only $18 \%$, which is the lowest in all cancers[1]. With widespread use of computed tomography (CT) in the clinic, the problems of small or faint lesions on CT become significant[2]. The resection of nodules by thoracoscopic surgery is difficult because the nodules may be hard to identify, especially for deep pulmonary small nodules. If the location of nodules cannot be accurately determined during the operation, it will increase the possibility of thoracotomy. Currently, preoperative localization of pulmonary nodules is widely used in the clinic, including CT-guided transthoracic approach, hook-wire and coil embolization, but these methods increase radiation exposure and cause complications[3].

Electromagnetic navigation bronchoscope can reach further peripheral lung compared to conventional electronic bronchoscope, and has a higher accuracy $[4,5]$. Combined with the path constructed by electronic navigation bronchoscope, we can mark the location of small pulmonary nodules. This method has better safety and effectiveness. Several studies have reported that electromagnetic navigation bronchoscopy (ENB) guided transbronchial needle biopsy achieved better accuracy and lower complication rates, compared to conventional percutaneous core needle biopsy[6-8]. In this study, we retrospectively compared ENB and CT-guided localization of small pulmonary nodules before resection.

\section{Methods}


We retrospectively analyzed patients who underwent localization with CT and ENB followed by Videoassisted thoracoscopic surgery (VATS) at Qilu Hospital of Shandong University from January 2016 to July 2019. Patients were included based on the following criteria: lung tumor requiring preoperative localization of only one pulmonary nodule, requiring surgical intervention according to the surgeon's judgment, and having undergone thoracoscopy after localization. Clinical parameters were recorded, including the gender, age, smoking status, histology, and stage. CT findings were recorded, including lesion size, location, density, and distance from pleural distance (PD). The nodule size was measured directly on CT images, and the longest diameter was the nodule size. The nodule was classified according to the density as pure nodular ground-glass opacity (GGO), 0-50\% GGO and >50\% GGO.

After general anesthesia with intubation, patients were navigated using the $7^{\text {th }}$ edition Super Dimension Navigation System (Covidien, Minneapolis, MN, USA) to localize and plan a route to the nodules one day before the operation. Thoracoscopic pulmonary surgery was performed immediately after the completion of magnetic navigation surgery.

\section{Percutaneous CT-Guided Localization}

CT scan was performed to confirm the presence of nodules before the localization. We used metal markers on the body surface to determine the missing line. Then we used CT and the laser to determine the coronal line. Coronal line represents the $\mathrm{CT}$ coronal plane of the lesion, and the intersection of two lines is used to determine the specific location of the nodule (Fig. 1).

\section{ENB planning and surgical procedures}

ENB-guided dye-marking was performed for subsolid nodules $<10 \mathrm{~mm}$ near the pleura or nodules $<20$ $\mathrm{mm}$ and located $>10 \mathrm{~mm}$ from the pleural surface. The axial, sagittal and coronal views of CT images were used for planning using super Dimension system (Medtronic, Minneapolis, MN, USA).The primary lung cancer and lymph nodes were dissected by VATS, and all patients received postoperative care. The clinicopathological data, procedure parameters, and complications were evaluated.

\section{Statistical Analyses}

Measurement data were presented as mean \pm standard deviation and analyzed by ANOVA. Count data were presented as percentage and analyzed by Chi-square test. Statistical analysis was performed using SPSS 23.0 software (SPSS Inc., Chicago, II, USA). P<0.05 was regarded as significance.

\section{Results}




\section{Characteristics of the patients}

Total 157 patients (88 males and 69 females) underwent the localization procedure successfully, and the nodules were localized by CT guidance in 105 patients and by ENB in 52 patients. Among 157 patients, 95 were smokers and 62 were non-smokers. Clinical characteristics of the patients and lung nodules were listed in Table 1. Each patient had only one nodule, and the 157 nodules included 74 pure GGO nodules, 66 part solid nodules with GGO $<50 \%$, and 17 part-solid nodules with GGO $>50 \%$. The nodule size was $6.99 \pm 1.37 \mathrm{~mm}$ (range $5.0-12.0 \mathrm{~mm}$ ). The nodule size of magnetic navigation localization was smaller than that of CT-guided localization. Magnetic navigation was more often applied in smaller nodules, while ENB was more often applied to joints near the lung surface. The nodules covered all the lobes, including 16 nodules in the left upper lobe, 26 nodules in the left lower lobe, 58 nodules in the right upper lobe, 17 nodules in the right middle lobe, and 40 nodules in the right lower lobe. The imaging diagnosis of 157 nodules was as follows: 29 benign lesion, 76 AIS, 44 MIA and 8 IA.

\section{Localization of the nodules}

Both CT-guided and ENB localization were well tolerated in all patients, without major complications or mortality. The incidence of asymptomatic hemopneumothorax was $7.6 \%(8 / 105)$, symptomatic hemopneumothorax was 3.8\% (4/105), hemoptysis was $0.9 \%$ (1/105) and decoupling was $0.9 \%(1 / 105)$ in CT-guided localization group. Under the same observation conditions, we did not find any complications in ENB group ( $P=0.107)$. In addition, in CT-guided localization group, 6 patients failed to be located while none failed in ENB group $(P=0.079)$. The procedure time for $C T$-guided localization was $15.15 \pm \llbracket 3.7 \mathrm{~min}$ (range 10-26 min), while the procedure time for ENB localization was $21.29 \pm \rrbracket 4.0 \mathrm{~min}$ (range 12-29 min) $(P<0.001)$. In CT-guided hookwire localization group, there were 6 hookwires pass through the nodule and 99 hookwires beside the nodule. The time of needle-carrying time for patients receiving CT-guided localization was $37.12 \pm 17.29 \min (P<0.001)$ (Table 2$)$.

\section{Discussion}

Because of the application of high resolution $\mathrm{CT}$, the rate of detecting small pulmonary lesions has increased. Therefore, it is important to accurately locate the sublobar or non-anatomical resection using a variety of approaches[9-12]. Currently used localization methods have several advantages and disadvantages. For example, percutaneous hook wire implantation has the advantages of simple operation, accurate location and less complications, but it still an invasive operation. Recently developed methods such as ENB could accurately access peripheral lung lesions beyond the reach of conventional bronchoscopy. In addition, ENB-guided location can reduce complications such as pneumothorax, hemothorax and hemoptysis, compared with traditional location methods. Therefore, ENB-guided location is regarded as a promising tool in thoracic surgery $[13,14]$. In this study we compared CT-guided localization and ENB-guided localization for lung nodules in patients with lung cancer.

In our study, 52 patients with magnetic navigation positioning had no complications, while 14 patients with CT-guided puncture hook positioning had complications. The most common complication was 
hemopneumothorax, but no catastrophic complications occurred. Magnetic navigation positioning technology has higher safety and operability, because all operations can be carried out in the operating room, while CT-guided positioning need a certain space distance and time interval, which increases the risk of whole positioning process. In addition, 6 (5.7\%) patients in CT-guided localization group failed to be located, mostly because of the deviation of the puncture hook position. Patients in ENB group need more time, because path setting has to be done before, and the operation of bronchoscopy is not particularly skilled for us. As the number of patients increases, the proficiency of ENB will be further improved, so we think that it is only a learning curve problem.

Puncture hook positioning method has additional problems. For example, when we complete the positioning, we usually cannot perform the operation immediately, and the patients need carry a puncture hook in the ward waiting for next surgical resection, which increases the complexity and risk of the operation. However, the procedure does not require additional facilities such as radiotracer, contrast injection or coil insertion. Therefore, CT-guided puncture hook positioning method is still simple and feasible, low cost, and widely used in clinical applications.

In contrast, navigational method has disadvantages of requiring much labor and experience to master and having low cost-effectiveness, but it has the advantages of small trauma, accurate location and safety. Moreover, ENB shows the potential for interventional lung surgery[13, 15-18]. Therefore, magnetic navigation and positioning technology has a good application prospect.

\section{List Of Abbreviations}

Computed Tomographic Imaging: CT; electromagnetic navigation bronchoscopy: ENB; Video-asssisted thoracoscopic surgery: VATS; distance from pleural distance: PD; ground-glass opacity (GGO)

\section{Declarations}

Ethics approval and consent to participate: This study was approved by the Ethics Committee of Qilu Hospital of Shandong University. The consent we obtained from study participants were written.

Consent for publication: The participates whose identifying images, CT scan images and specimen picture were used in this article provided the constant for publication.

Availability of data and materials: The datasets used and/or analysed during the current study are available from the corresponding author on reasonable request.

Competing interests: The authors declare that they have no competing interests.

Funding: No funding was obtained for this study. 
Authors' contributions: TH designed research; TY, LM, YWM and SLB conducted research; LM, YWM and LSH analyzed data; TY and LL wrote the paper; TH had primary responsibility for final content. All authors have read and approved the manuscript.

Acknowledgements: Not Applicable.

\section{References}

1. Siegel RL, Miller KD, Jemal A: Cancer statistics, 2018. CA Cancer J Clin 2018, 68(1):7-30.

2. Rubin GD: Lung nodule and cancer detection in computed tomography screening. $J$ Thorac Imaging 2015, 30(2):130-138.

3. Park CH, Han K, Hur J, Lee SM, Lee JW, Hwang SH, Seo JS, Lee KH, Kwon W, Kim TH et al: Comparative Effectiveness and Safety of Preoperative Lung Localization for Pulmonary Nodules: A Systematic Review and Meta-analysis. Chest 2017, 151(2):316-328.

4. Arenberg D: Electromagnetic navigation guided bronchoscopy. Cancer Imaging 2009, 9:89-95.

5. Seijo LM: Electromagnetic navigation bronchoscopy: clinical utility in the diagnosis of lung cancer. Lung Cancer (Auckl) 2016, 7:111-118.

6. Arias S, Lee H, Semaan R, Frimpong B, Ortiz R, Feller-Kopman D, Oakjones-Burgess K, Yarmus L: Use of Electromagnetic Navigational Transthoracic Needle Aspiration (E-TTNA) for Sampling of Lung Nodules. J Vis Exp 2015(99):e52723.

7. Narsule CK, Sales Dos Santos R, Gupta A, Ebright MI, Rivas R, Jr., Daly BD, Fernando HC: The efficacy of electromagnetic navigation to assist with computed tomography-guided percutaneous thermal ablation of lung tumors. Innovations (Phila) 2012, 7(3):187-190.

8. Makris D, Scherpereel A, Leroy S, Bouchindhomme B, Faivre JB, Remy J, Ramon P, Marquette CH: Electromagnetic navigation diagnostic bronchoscopy for small peripheral lung lesions. Eur Respir $J$ 2007, 29(6):1187-1192.

9. Lee NK, Park CM, Kang CH, Jeon YK, Choo JY, Lee HJ, Goo JM: CT-guided percutaneous transthoracic localization of pulmonary nodules prior to video-assisted thoracoscopic surgery using barium suspension. Korean J Radiol 2012, 13(6):694-701.

10. Bellomi M, Veronesi G, Trifiro G, Brambilla S, Bonello L, Preda L, Casiraghi M, Borri A, Paganelli G, Spaggiari L: Computed tomography-guided preoperative radiotracer localization of nonpalpable lung nodules. Ann Thorac Surg 2010, 90(6):1759-1764.

11. Kondo R, Yoshida K, Hamanaka K, Hashizume M, Ushiyama T, Hyogotani A, Kurai M, Kawakami S, Fukushima M, Amano J: Intraoperative ultrasonographic localization of pulmonary ground-glass opacities. J Thorac Cardiovasc Surg 2009, 138(4):837-842.

12. Doo KW, Yong HS, Kim HK, Kim S, Kang EY, Choi YH: Needlescopic resection of small and superficial pulmonary nodule after computed tomographic fluoroscopy-guided dual localization with radiotracer and hookwire. Ann Surg Oncol 2015, 22(1):331-337. 
13. Munoz-Largacha JA, Ebright MI, Litle VR, Fernando HC: Electromagnetic navigational bronchoscopy with dye marking for identification of small peripheral lung nodules during minimally invasive surgical resection. J Thorac Dis 2017, 9(3):802-808.

14. Pearlstein DP, Quinn CC, Burtis CC, Ahn KW, Katch AJ: Electromagnetic navigation bronchoscopy performed by thoracic surgeons: one center's early success. Ann Thorac Surg 2012, 93(3):944-949; discussion 949-950.

15. Xie F, Zheng X, Xiao B, Han B, Herth FJF, Sun J: Navigation Bronchoscopy-Guided Radiofrequency Ablation for Nonsurgical Peripheral Pulmonary Tumors. Respiration 2017, 94(3):293-298.

16. Banovac F, Cheng P, Campos-Nanez E, Kallakury B, Popa T, Wilson E, Abeledo H, Cleary K: Radiofrequency ablation of lung tumors in swine assisted by a navigation device with preprocedural volumetric planning. J Vasc Interv Radiol 2010, 21(1):122-129.

17. Amalou $\mathrm{H}$, Wood $\mathrm{BJ}$ : Electromagnetic tracking navigation to guide radiofrequency ablation of a lung tumor. J Bronchology Interv Pulmonol 2012, 19(4):323-327.

18. Zhao ZR, Lau RWH, Ng CSH: Catheter-based alternative treatment for early-stage lung cancer with a high-risk for morbidity. J Thorac Dis 2018, 10(Suppl 16):S1864-S1870.

\section{Tables}

Table 1 Clinical characteristics and demographics of two matched groups. 


\begin{tabular}{|c|c|c|c|}
\hline Variables & CT-Guided Localization & ENB & $P^{*}$ \\
\hline age & & & 0.459 \\
\hline$>60$ & 51 & 22 & \\
\hline$<60$ & 54 & 30 & \\
\hline Gender & & & 0.527 \\
\hline Male & 57 & 31 & \\
\hline Female & 48 & 21 & \\
\hline Smoking & & & 0.116 \\
\hline No & 46 & 16 & \\
\hline Yes & 59 & 36 & \\
\hline Location & & & 0.066 \\
\hline RUL & 44 & 14 & \\
\hline RML & 11 & 6 & \\
\hline RLL & 20 & 20 & \\
\hline LUL & 13 & 3 & \\
\hline LLL & 17 & 9 & \\
\hline Nodule size & & & $<0.001$ \\
\hline$<6 \mathrm{~mm}$ & 8 & 11 & \\
\hline 6-8mm & 43 & 38 & \\
\hline$>8 \mathrm{~mm}$ & 54 & 3 & \\
\hline Nodule depth & & & 0.007 \\
\hline $0 \mathrm{~cm}$ & 3 & 0 & \\
\hline $0-3 \mathrm{~cm}$ & 61 & 43 & \\
\hline$>3 \mathrm{~cm}$ & 41 & 9 & \\
\hline
\end{tabular}




\begin{tabular}{lccc} 
Nodule density & & & 0.567 \\
Pure GGO & 47 & 27 & \\
$>50 \% G G O$ & 45 & 21 & \\
$<50 \% G G O$ & 13 & 4 & \\
Pathological Diagnosis & & & 0.117 \\
Benign lung tumor & 15 & 14 & \\
AIS & 50 & 26 & \\
MIA & 33 & 11 & \\
IA & 7 & 1 \\
\hline
\end{tabular}

* Chi-square test

Table 2 Localization and surgery results for the ENB and CT groups.

\begin{tabular}{llll}
\hline Variables & CT-Guided Localization & ENB & $P^{\star}$ \\
\hline Complications & & & 0.107 \\
No complications & 91 & 52 & \\
Asymptomatic hemopneumothorax & 8 & 0 & \\
Symptomatic hemopneumothorax & 4 & 0 & \\
Hemoptysis & 1 & 0 & \\
Decoupling & 1 & 0 & \\
Failed to localization & 6 & 0 & 0.079 \\
Localization time(min) & $15.15 \pm \square 3.7$ & $21.29 \pm \square 4.0$ & $<0.001$ \\
Hookwire station & & & - \\
$\quad$ Through the nodule & & & \\
Beside the nodule & 6 & - & $<0.001$ \\
Needle-carrying time(min) & 99 & 0 & \\
\hline
\end{tabular}

* Chi-square test

\section{Figures}


A

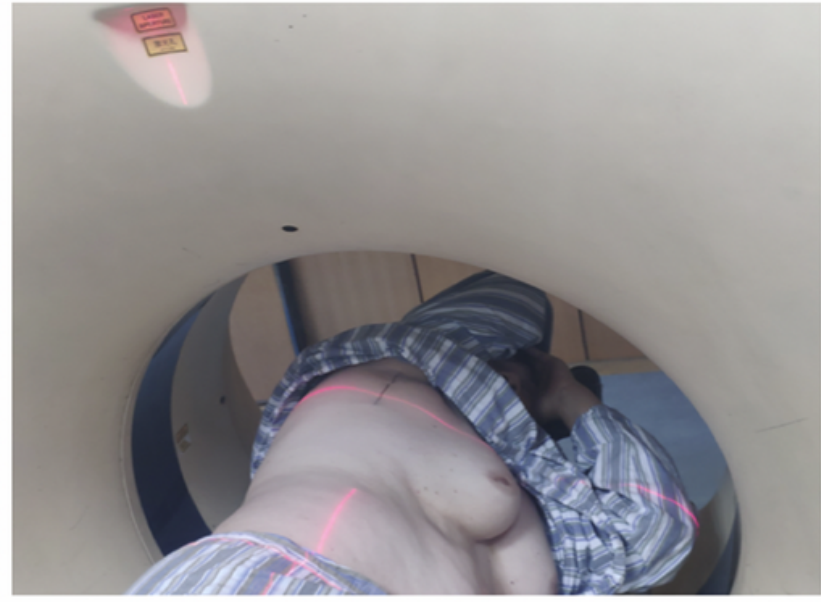

C

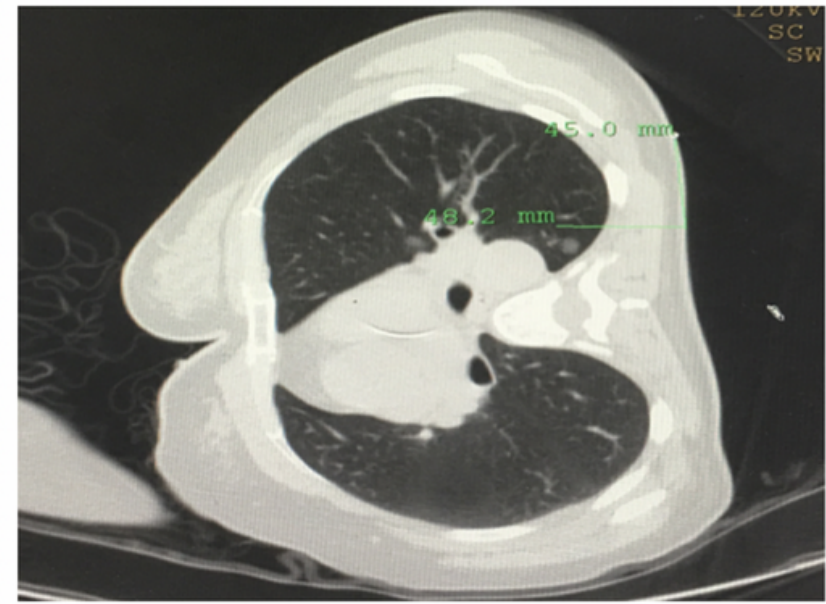

B
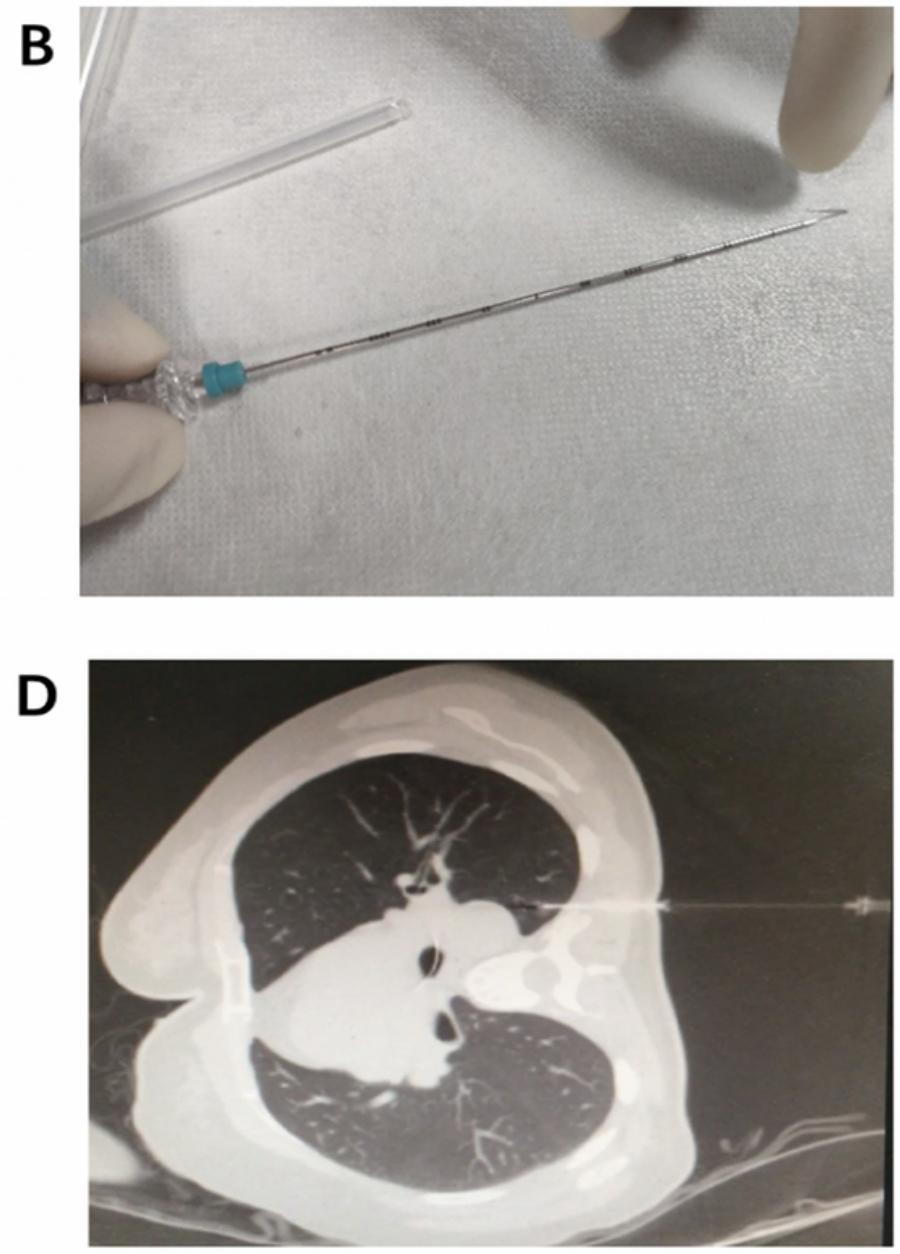

\section{Figure 1}

(A) Coronal location by CT and Sagittal marking with steel needles. (B). The picture of steel needle. (C) Measuring the distance of puncture point and locating the distance between steel needle and puncture point in coronal position of CT image. (D) The results of puncture were confirmed by CT and the complications were detected. 

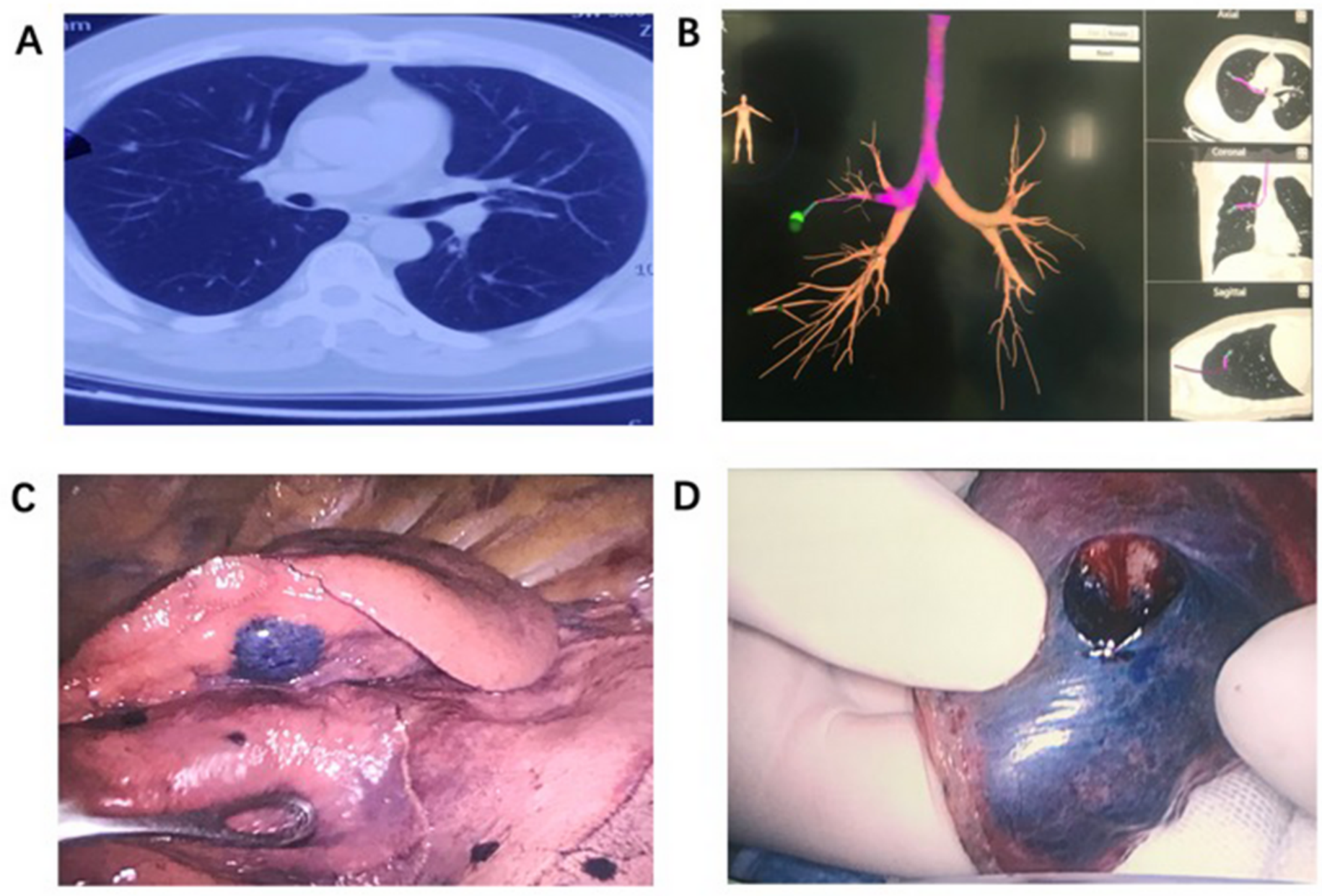

\section{Figure 2}

(A) A small pulmonary nodule is located in the right upper lobe. (B)Before operation, magnetic navigation software was used to design magnetic navigation location path. (C) According to the designed path, the magnetic navigation probe was stained with methylene blue after reaching the lesion location. (D) The accuracy of localization was verified after thoracoscopic surgery. 
A

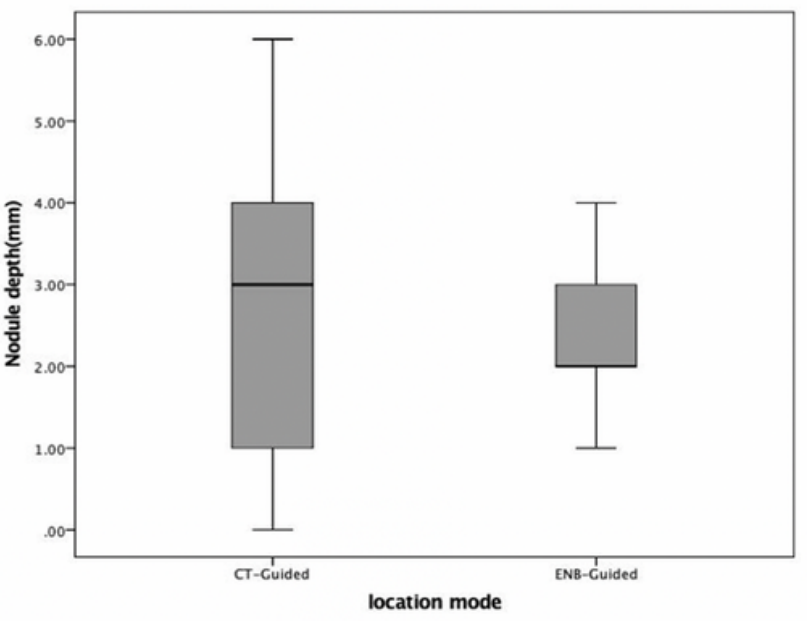

C

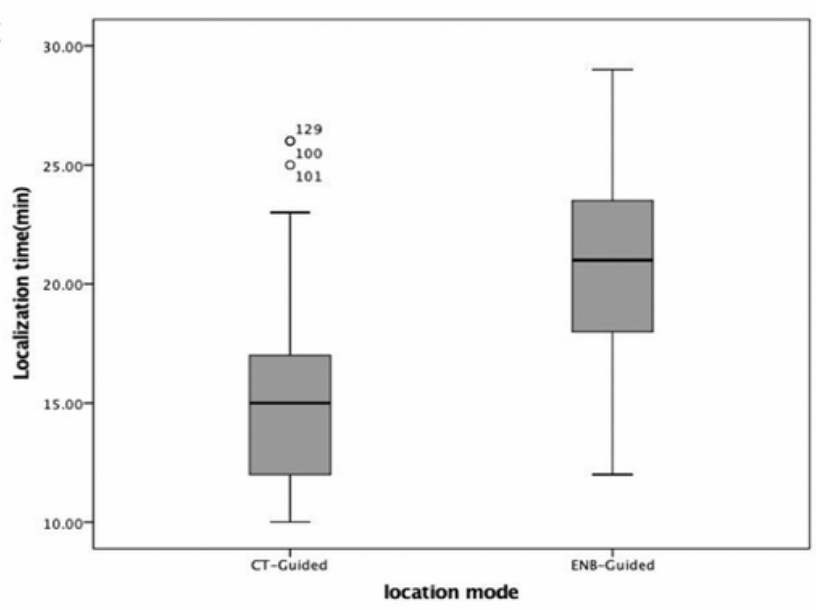

B

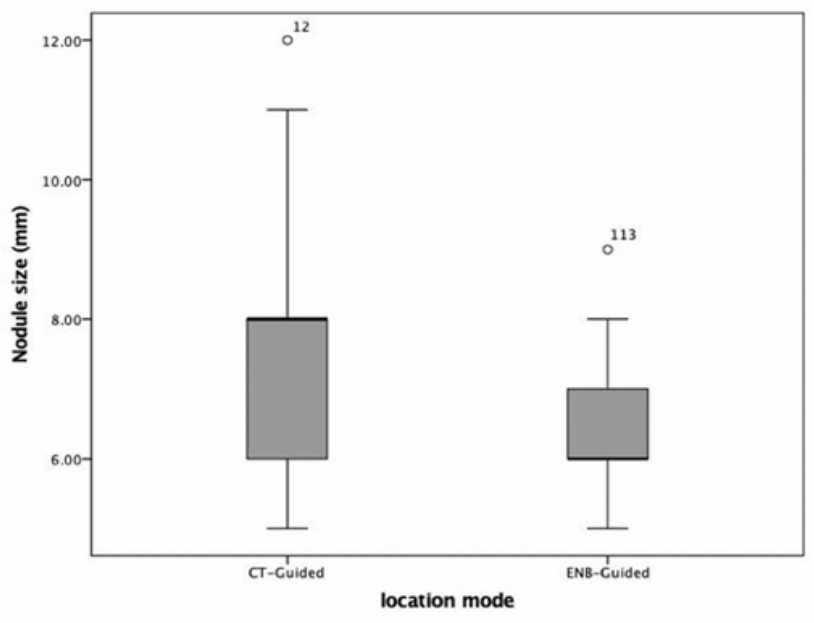

\section{Figure 3}

Characteristics of 107 pulmonary nodular lesion of two matched groups. (A)Nodule depth. (B) Nodule size (C) Localization time. 